\title{
Persistence of ferroelectricity above the Curie temperature at the surface of $\mathrm{Pb}\left(\mathrm{Zn}_{1 / 3} \mathrm{Nb}_{2 / 3}\right) \mathrm{O}_{3}-12 \% \mathrm{PbTiO}_{3}$
}

\author{
N. Domingo, ${ }^{1}$ N. Bagués, ${ }^{1,2}$ J. Santiso,,${ }^{1,2}$ and G. Catalan ${ }^{1,3, *}$ \\ ${ }^{1}$ ICN2 - Institut Catala de Nanociencia i Nanotecnologia, Campus UAB, 08193 Bellaterra (Barcelona), Spain \\ ${ }^{2}$ CSIC - Consejo Superior de Investigaciones Cientificas, ICN2 Building, 08193 Bellaterra (Barcelona), Spain \\ ${ }^{3}$ ICREA - Institucio Catalana de Recerca i Estudis Avançats, 08010 Barcelona, Spain
}

(Received 21 July 2014; revised manuscript received 17 December 2014; published 26 March 2015)

\begin{abstract}
Relaxor-based ferroelectrics have been known for decades to possess a relatively thick surface layer ("skin") that is distinct from its interior. Yet while there is consensus about its existence, there are controversies about its symmetry, phase stability, and origin. In an attempt to clarify these issues, we have examined the surface layer of PZN-12\%PT. While the bulk transitions from a ferroelastically twinned tetragonal ferroelectric state with in-plane polarization to a cubic paraphase at $T_{\mathrm{c}}=200^{\circ} \mathrm{C}$, the skin layer shows a robust labyrinthine nanodomain structure with out-of-plane polarization that persists hundreds of degrees above the bulk Curie temperature. Cross-sectional transmission electron microscopy analysis shows that the resilience of the skin's polarization is correlated with a compositional imbalance: lead vacancies at the surface are charge-compensated by niobium enrichment; the excess of $\mathrm{Nb}^{5+}$-a small ion with $d^{0}$ orbital occupancy—stabilizes the ferroelectricity of the skin layer.
\end{abstract}

DOI: 10.1103/PhysRevB.91.094111

PACS number(s): 77.80.B-, 77.80.Dj, 68.37.Ps, 68.35.Dv

Morphotropic phase boundary relaxors have been intensely studied since the discovery in 1997 of their giant electromechanical performance [1]. Even before this finding, relaxors had already intrigued physicists for decades [2,3] on account of their unusual dielectric properties, characterized by a broad dielectric peak whose maximum is frequency dependent, indicative of a relaxation process rather than a sharp phase transition-hence the name "relaxors." The relaxation dynamics have traditionally been linked to the existence of chemical and structural heterogeneity on a nanoscopic scale, with random bonds and random fields disrupting ferroelectric ordering [4-6] and leading to a glassy transition characterized by Vogel-Fulcher freezing of the polar dynamics [7]. In solid solutions with conventional ferroelectrics, and depending on the relative content of the relaxor [the archetypes being $\mathrm{Pb}\left(\mathrm{Mg}_{1 / 3} \mathrm{Nb}_{2 / 3}\right) \mathrm{O}_{3}$ and $\left.\mathrm{Pb}\left(\mathrm{Zn}_{1 / 3} \mathrm{Nb}_{2 / 3}\right) \mathrm{O}_{3}\right]$ and ferroelectric (such as $\mathrm{PbTiO}_{3}$ ), a rich phase diagram emerges, with the salient feature being a morphotropic phase boundary where polar rotation enhances the electromechanical response $[8,9]$.

An intriguing feature of relaxor ferroelectrics is that they possess fairly thick surface layers ("skin layers") with different structure [10-12] and lower permittivity [13] compared to bulk. Subsequent investigations by piezoresponse force microscopy have shown that the surface possesses a mesoscopic (submicron) labyrinthine domain structure [14-18]. Questions and controversies surround this surface layer. While initial evidence suggested that the skin had different symmetry from the bulk [19], later studies by Kisi and Forrester [20] detected no difference in symmetry, prompting the authors to suggest that the difference between bulk and surface may just be in the domain state. The surface domains have themselves been variously linked to polishing stress [14] and to the symmetry-breaking effect of the surface on the ordering of

*gustau.catalan@cin2.es relaxor polar nanoregions [14-18]. There is no consensus about the thickness of the surface layer, nor about whether its phase transitions coincide with those of the bulk. It is the purpose of this paper to shed light on these questions, and determine not only the structural characteristics of the surface layer but also the link between them and the polar behavior of the surface, which we have found to remain ferroelectric well above the bulk Curie temperature.

Single crystals of $\mathrm{Pb}\left(\mathrm{Zn}_{1 / 3} \mathrm{Nb}_{2 / 3}\right) \mathrm{O}_{3}-12 \% \mathrm{PbTiO}_{3}$, hereafter labeled as PZN-12\%PT, were commercially acquired from Microfine Materials Technologies Pte, Ltd. The perovskite crystals were (100)-oriented, and x-ray diffraction confirmed the crystals to be tetragonal at room temperature. The asreceived crystals had optically smooth surfaces obtained by mechanical polishing using forces of the order of millinewtons. The local properties of the surfaces were tested using an atomic force microscope (AFM), model MFP-3D from Asylum Research. An optical microscope attached to the AFM head allowed monitoring the macroscopic (bulk) domain structure simultaneously with scanning probe measurements. Conductive tips from Nanosensors model EFM (2 N/m, PtIr coating) were used for the characterization of the ferroelectric domains in piezoresponse force microscopy (PFM) mode. PFM measurements, which detect the expansion and contraction of a sample under an ac voltage applied through the tip working in contact mode [21-23], were done at the contact resonance frequency using dual-amplitude resonance tracking (DART) [24]. Temperature control between room temperature and $300{ }^{\circ} \mathrm{C}$ was afforded using Asylum's PolyHeater stage. The structural properties of the surface and the bulk were measured by standard and grazing-incidence x-ray diffraction, using a PANalytical X'Pert Pro diffractometer with fourcircle goniometer equipped with an Anton-Paar DHS1100 hot stage. In addition, we used cross-sectional transmission electron microscopy (TEM) (Tecnai F20, FEI) to map the local microstructure and the compositional balance across the thickness of the crystal. 

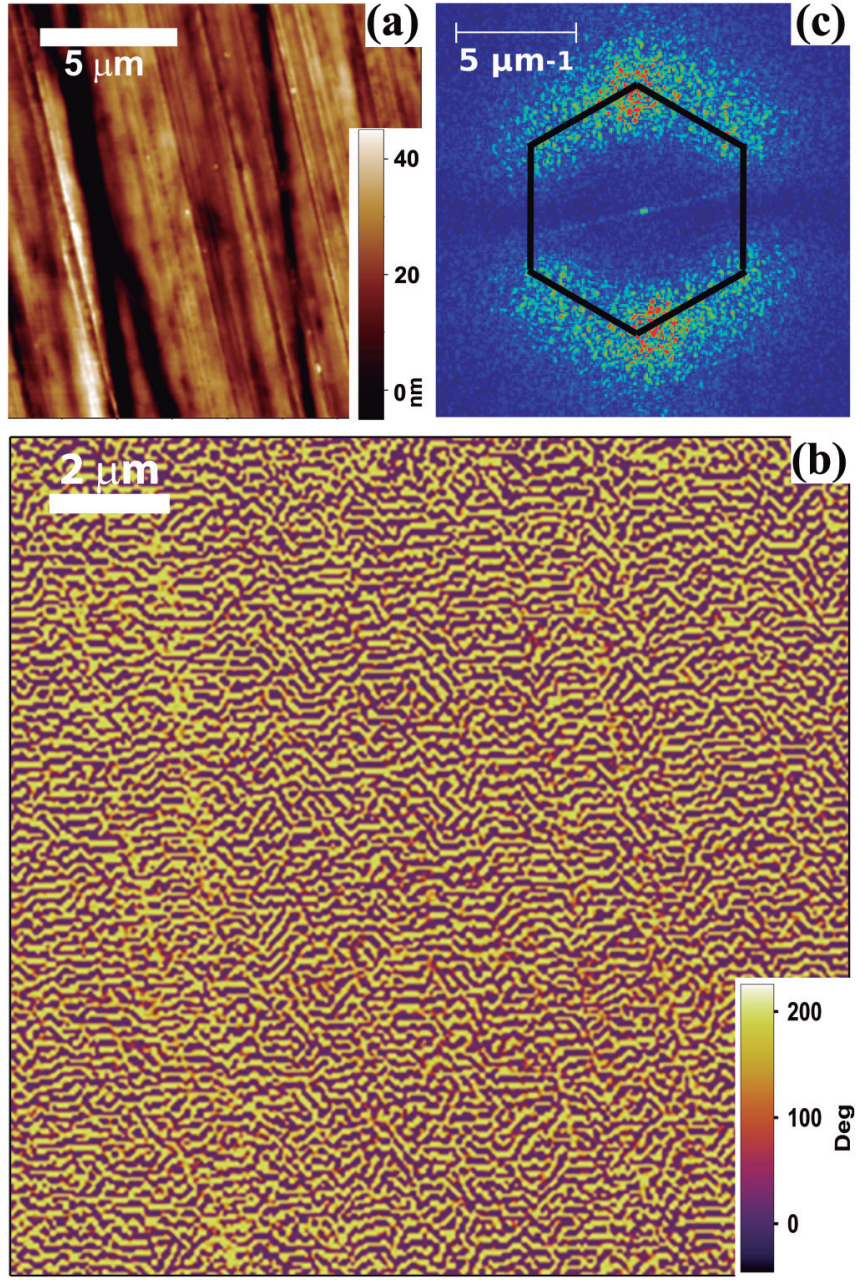

FIG. 1. (Color online) (a) Topography AFM image of the surface of PZN-12\%PT single crystals of an area of $15 \times 15 \mu \mathrm{m}$ and (b) the corresponding phase image of VPFM of the same area. (c) Fast Fourier transform of the VPFM phase image, showing a hexagonal symmetry with a periodicity of $\sim 190 \mathrm{~nm}$.

Figure 1 shows a PFM scan of the ferroelectric domain structure at the surface, with a regular periodicity of $190 \pm 50 \mathrm{~nm}$ (domain width $95 \mathrm{~nm}$ ), running along six preferred directions of the plane, as shown by the fast Fourier transform of the amplitude of the vertical piezoresponse force microscopy (VPFM) image [Fig. 1(c)]. Lateral PFM (not shown) confirms that the polarization of the domains is out of plane; in other words, the labyrinthine pattern corresponds to 180-degree domains of polarization perpendicular to the surface. This is consistent with the meandering aspect of the domain walls, as 180-degree domains are not subject to stringent geometric constraints in the in-plane direction [25]. As for the tendency towards hexagonal arrangement, it may be caused by a weak crystallographic preference for some planes, by a close-packing trend, or by a recently discovered faceting instability of nanodomains [26]. We note in any case that such arrangements are ubiquitous in thin films of very different physical systems, ranging from flux patterns in superconductors and 180-degree domains in ferromagnets to chemically segregated films of lipids, liquid crystals, or block copolymers [27-29].
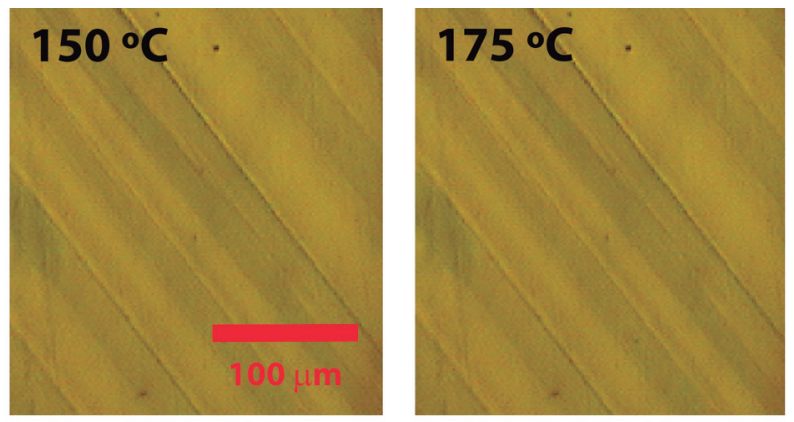

(a)
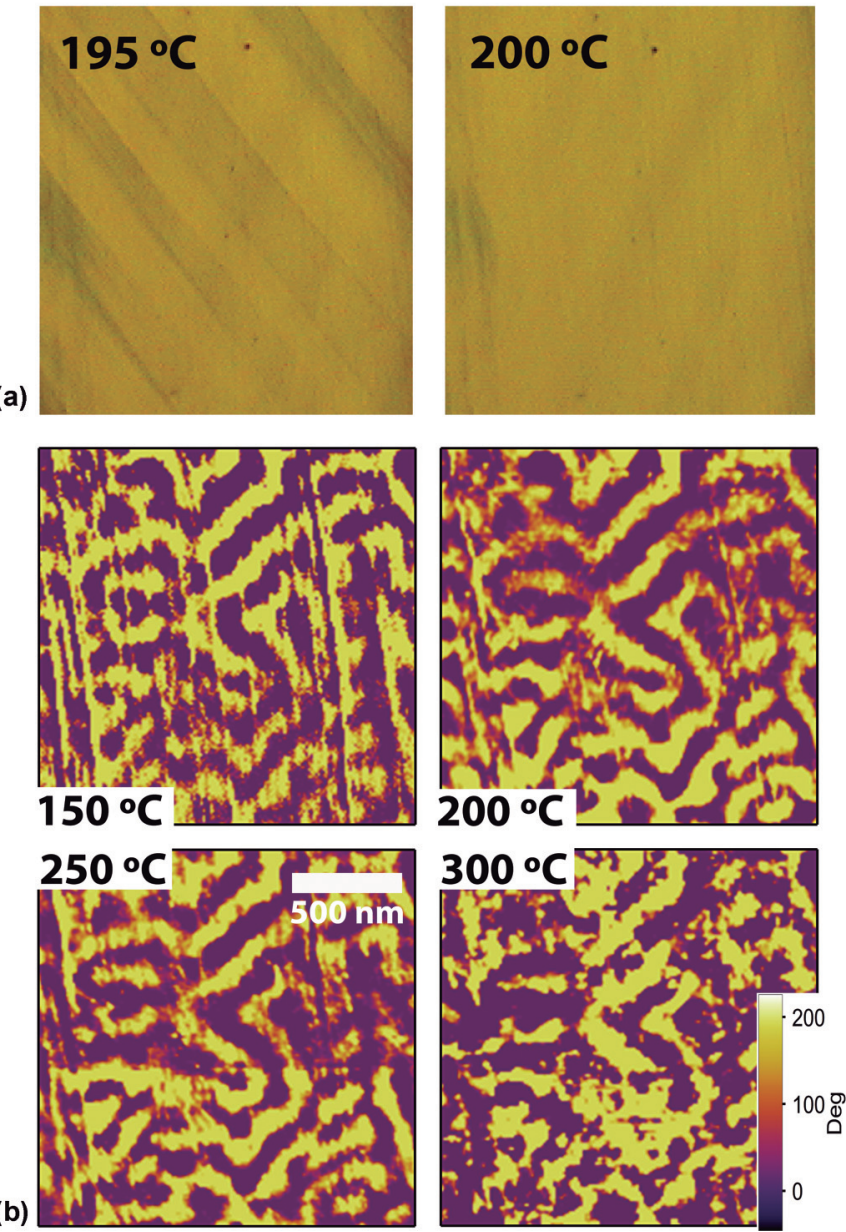

FIG. 2. (Color online) (a) Optical microscopy images of the bulk domains of PZN-12\%PT single crystals. Even when the light is not polarized it is still possible to distinguish the ferroelastic domain walls present in the single crystal. At $195^{\circ} \mathrm{C}$ the bulk domains start disappearing and they are completely removed at $200^{\circ} \mathrm{C}$. (b) Phase image of VPFM of an area of $1.8 \times 1.8 \mu \mathrm{m}$ of PZN-12\%PT at 150 , 200,250 , and $300^{\circ} \mathrm{C}$. The surface domain structure remains identical across and above the bulk phase transition, persisting up to $300^{\circ} \mathrm{C}$, the highest temperature achievable of our sample heater in the AFM.

PFM is intrinsically a surface probe that cannot access the interior of a crystal. For a view of the interior, we use optical microscopy (Fig. 2) and x-ray diffraction (Fig. 3). Figure 2 shows the temperature dependence of the domain structure, as observed both in transmission optical microscopy and in PFM. The straight lines running diagonally across the optical micrographs correspond to 90-degree ferroelastic twins, in 

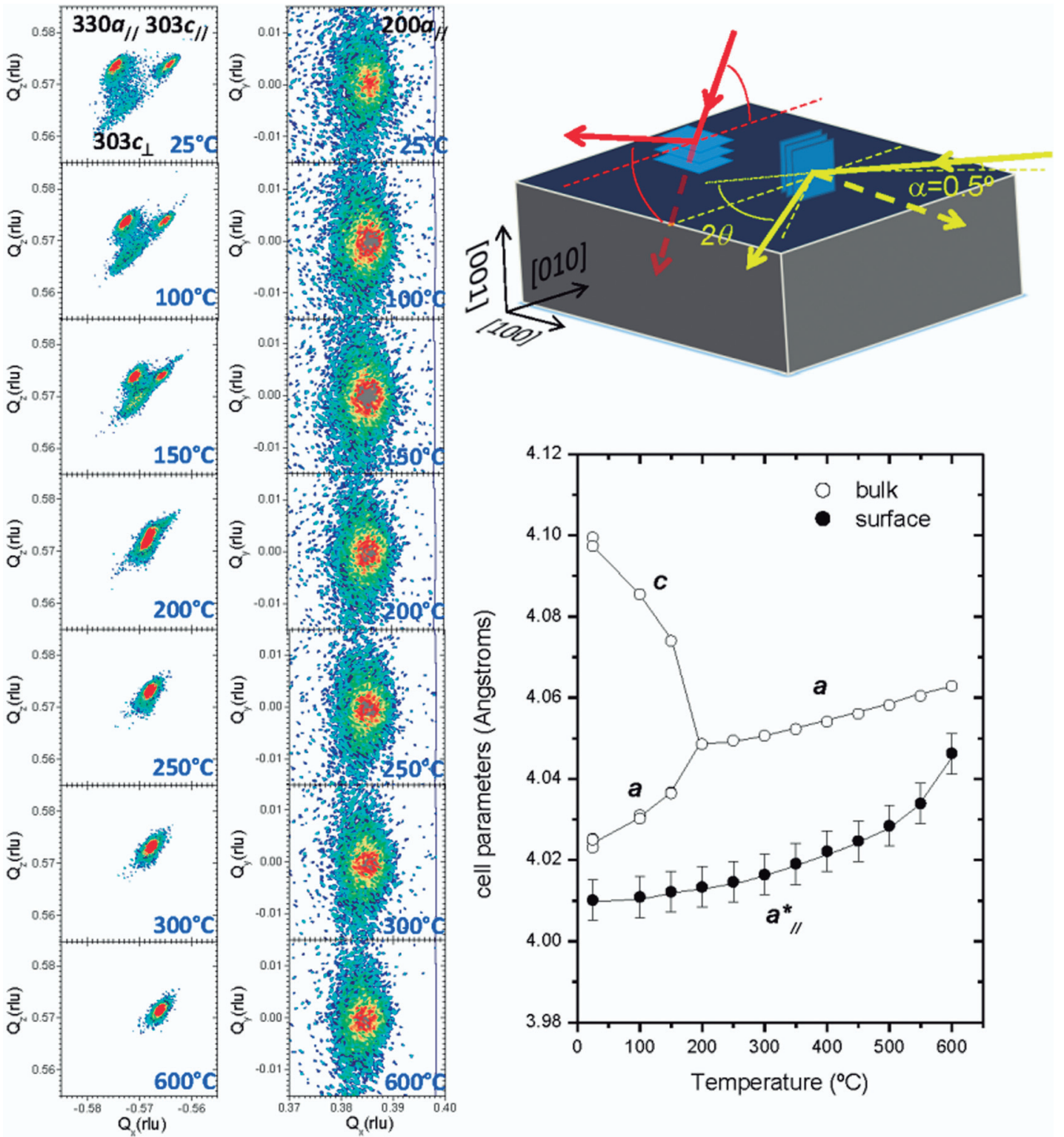

FIG. 3. (Color online) (Left) X-ray diffraction $Q_{\mathrm{x}}-Q_{\mathrm{z}}$ reciprocal space maps of the (303) region of the bulk crystal at different temperatures from room temperature to $600{ }^{\circ} \mathrm{C}$, along with in-plane $Q_{\mathrm{x}}-Q_{\mathrm{y}}$ maps of the 200 surface reflection measured in grazing incidence and exit angles $\left(\alpha=0.5^{\circ}\right)$. (Right) Scheme of both measurement geometries. Bulk measurement shows several reflections corresponding to the expected tetragonal twinning, while the surface shows a single reflection in the whole temperature range explored. Calculated lattice parameters from $\mathrm{x}$-ray diffraction as a function of temperature, showing the disappearance of the tetragonal twinning at $200{ }^{\circ} \mathrm{C}$ (bulk Curie temperature) and the nonlinear thermal expansion of the surface, suggesting a possible phase transition just above 600 Celsius.

accordance with the bulk tetragonal symmetry of PZN-12\%PT at room temperature [30]. At $T_{\mathrm{c}}=200^{\circ} \mathrm{C}$, the ferroelastic twins abruptly disappear, signaling the transition to the cubic paraelectric phase in the bulk of the crystal. In contrast, the labyrinthine domains observed by PFM remain unaltered up to at least $300^{\circ} \mathrm{C}$, which is the highest temperature achievable in our AFM. The labyrinthine domains are therefore decoupled from the bulk Curie temperature.

In order to gain further insight on the lattice structure at different depths within the crystal, we combine grazingincidence $\mathrm{X}$-ray diffraction [31] (at incidence angle $\alpha=$ $0.5^{\circ}$ ) and standard Bragg-Brentano x-ray diffraction (large incidence angle): the former has a penetration depth $<100 \mathrm{~nm}$, while the latter penetrates to several microns. The results are shown in Fig. 3. The bulk-sensitive $Q_{\mathrm{x}} Q_{\mathrm{z}}$ reciprocal space map around the (303) primitive reflection shows two intense spots and a much weaker one. The $Q_{\mathrm{z}}$ coordinate of the intense reflections is the same for both and consistent with the expected length of the $a$-axis parameter, while their different $Q_{\mathrm{x}}$ coordinates indicate that one corresponds to the $a$ axis and $c$ axis of the PZN-PT tetragonal phase. Therefore, these reflections correspond to twin domains alternating polarization along the [100] and [010] axes (orthogonal directions in the plane of the crystal). The maps show also a weaker reflection corresponding to a much smaller fraction of $c_{\perp}$-oriented domains. 
Accurate determination of the cell parameters at room temperature results in a tetragonal phase with $a=4.02( \pm 0.01) \AA$ and $c=4.08( \pm 0.01) \AA$. Meanwhile, at grazing incidence (surface-sensitive), there is only one (200) spot corresponding to a relatively short in-plane lattice parameter of 4.00 $( \pm 0.01) \AA$. (Note that in the symmetric geometry of our in-plane measurement, cell parameter determination is not affected by refraction shifts.) We also performed grazingincidence scans around the (110) reflection and saw no evidence of splitting, ruling out orthorhombic or rhombohedral twinning. The surface diffraction thus indicates a square inplane lattice parameter with no twinning, while the PFM results indicate that the polar axis is out of plane. The skin results are therefore consistent with a tetragonal lattice with $c$ out of plane; we nevertheless note that although this is the highest symmetry consistent with our results, a monoclinic phase with no twinning and pure 180-degree domains cannot be excluded by these measurements.

Diffraction as a function of temperature, shown in Fig. 3, also confirms that the surface is structurally decoupled from the interior: while the bulk tetragonal splitting decreases and disappears at $T_{\mathrm{c}}=200^{\circ} \mathrm{C}$, the single-peaked skin layer shows no phase transition all the way up to $600^{\circ} \mathrm{C}$, which is the maximum temperature reached in the measurements. It is nevertheless worth noticing that the rate of thermal expansion of this surface lattice parameter is not linear, and its shape suggests the existence of a phase transition at an extrapolated critical temperature just above $600^{\circ} \mathrm{C}$.

In order to clarify the morphology and stoichiometry of the skin layer, we turn to cross-sectional transmission electron microscopy. Cross-sectional TEM (Fig. 4) uncovers three important pieces of information. First, there is a clear structural discontinuity at a depth of ca. $0.5 \mu \mathrm{m}$. This is therefore the thickness of the skin layer in our crystals. Second, the skin is considerably "tortured" in comparison with the bulk: simple visual inspection shows the density of dislocations to be bigger, and electron diffraction (inset) confirms the existence of considerable mosaicity; both observations are consistent with stress-induced changes due to sample polishing, as proposed by Wong and Zeng [14,15]. We parenthetically note that polishing stress has been shown to induce the appearance of polar modes in otherwise centrosymmetric materials such as $\mathrm{CaWO}_{4}$ and $\mathrm{CaMoO}_{4}[32]$.

The third and most important observation is that there is a compositional variation as a function of depth, as measured by energy-dispersive $\mathrm{x}$-ray analysis (EDX) and shown in Fig. 4. The skin layer is found to be lead-deficient, perhaps due to the high volatility of lead. In order to preserve charge neutrality, each $\mathrm{Pb}^{2+}$ vacancy could in theory be compensated by an $\mathrm{O}^{2-}$ vacancy. Instead, however, we find that the loss of $\mathrm{Pb}^{2+}$ is neutralized by an excess of $\mathrm{Nb}^{5+}$ : the average ratio of $\mathrm{Nb} / \mathrm{Pb}$ is markedly greater at the surface than in the interior. The displacement of ions required for this imbalance would normally be associated with high temperatures, where ionic mobility is higher, whereas our samples are polished at room temperature. We note, however, that polishing is by definition an aggressive process capable of removing material from the surface; our results suggest that it can also move ions below it.
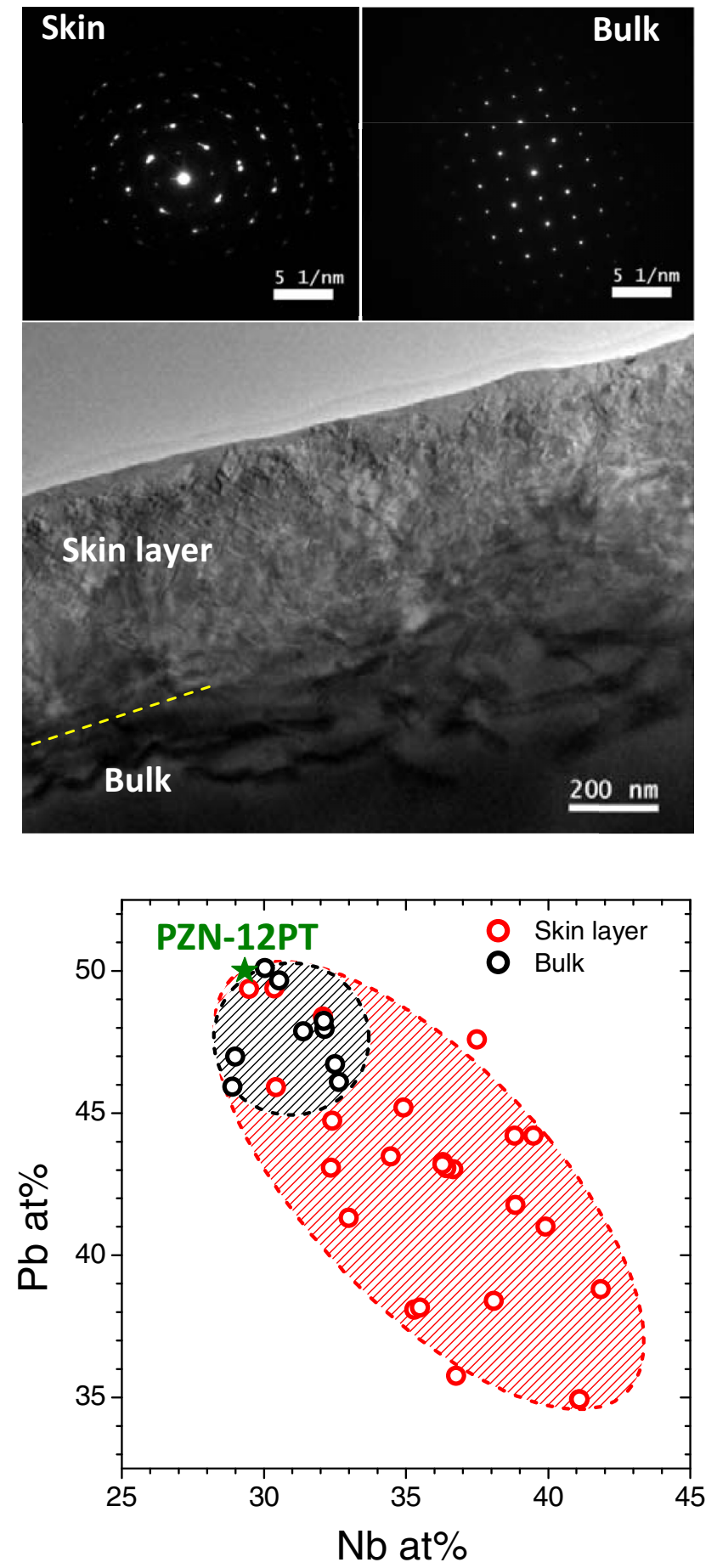

FIG. 4. (Color online) (Above) Cross-sectional TEM image close to the PZN-PT crystal surface area showing a distinct skin layer with higher density of dislocations, along with corresponding electron diffraction patterns (top) of the bulk and surface regions, showing remarkably different mosaicity. (Below) $\mathrm{Pb}$ versus $\mathrm{Nb}$ at $\%$ composition measured by EDX across the bulk, intermediate, and surface regions of the skin layer. Notice the lead loss compensated by $\mathrm{Nb}$ excess across the skin layer.

The excess niobium has profound consequences for the polarization of the surface. In lead-based ferroelectrics, the lone pair of the $\mathrm{Pb}^{+2}$ ion at the $A$ site has an important contribution to the ferroelectricity [33], but in relaxor solid 
solutions, ionic size and $d^{0}$ orbital occupancy at the B site ultimately determine the critical temperature, which is highest for pure $\mathrm{PbTiO}_{3}$ (pure $d^{0}$ orbital occupancy at the $\mathrm{B}$ site) and lowest for pure PZN, where only $1 / 3$ of the B sites are occupied by $d^{0}$ ions. The key role of $\mathrm{Nb}^{5+}$ as "polarization carrier" in PZN has been identified by Al-Zein et al. [34]: $\mathrm{Nb}^{5+}$ has $d^{0}$ orbital occupancy, which favors ferroelectricity through orbital hybridization with the oxygen ions [33]. In addition, $\mathrm{Nb}^{5+}$ is a much smaller ion $(r=0.64 \AA$ [35]) than $\mathrm{Zn}^{2+}(0.74 \AA)$, being close in size to $\mathrm{Ti}^{4+}(0.605 \AA)$. Excess $\mathrm{Nb}^{5+}$ thus acts similarly to doping with $\mathrm{Ti}^{4+}$ : it stabilizes the ferroelectric phase and increases the local $T_{\mathrm{c}}$, with the bigger charge and ionic polarizability [36] of $\mathrm{Nb}^{5+}$ compared to $\mathrm{Zn}^{2+}$ or $\mathrm{Ti}^{4+}$ further contributing to the polarization enhancement.

The results are reminiscent of those reported for multiferroic $\mathrm{BiFeO}_{3}$, for which a skin layer was also found with its own phase transitions and domain structure independent from the bulk's [36-38]. In common with $\mathrm{BiFeO}_{3}$, relaxor-based ferroelectrics have an energy landscape with low energy barriers between competing phases [39] and, also like in $\mathrm{BiFeO}_{3}$, the $A$-site ion is highly volatile, leading to surface vacancies that can alter the phase equilibrium [37]. Unlike in $\mathrm{BiFeO}_{3}$ (BFO), however, the $A$-site vacancies of PZN-PT can be compensated by changing the stoichiometry of the ions in the $\mathrm{B}$ site, resulting in an excess of small and highly polarizable $\mathrm{Nb}$ ions that stabilize ferroelectricity. As a consequence, while in BFO the skin displays more temperature-driven phase transitions than the bulk [37-39], in PZN-PT the converse is true, with the tetragonal polar phase being chemically stabilized up to at least $600^{\circ} \mathrm{C}$.

Morphotropic phase boundary relaxors are used as the transducing elements in high-end applications [40] and as active substrates in dynamic strain-tuning of oxide thin films [41]; since electromechanical coupling is mediated by the interface, the surface piezoelectricity will be an integral part of the device performance. On a more fundamental level, the results raise broader questions about the impact of the surface layer on the properties of relaxor ferroelectrics, in particular, with regard to their well-documented polar activity (e.g., birefringence) at high temperatures [42]. Traditionally, this birefringence has been attributed to polar nanoregions inside the crystals, but such a view is still debated [43], and the persistence of ferroelectricity in a thick skin layer has consequences for the overall polar activity of the crystals that should be incorporated into the analysis.

We acknowledge financial support from the Spanish Ministerio de Ciencia e Innovación (Contracts No. MAT201017771, No. MAT2011-29081-C02, and No. FIS2013-48668C2-1-P), and the Generalitat de Catalunya (Project 2014 SGR 1216). N.D. thanks the Spanish Ministerio de Ciencia e Innovación for the Ramon y Cajal Research Grant (No. RYC2010-06365), and G.C. acknowledges an ERC Starting Grant (Project reference: ERC-SG-308023). ICN2 acknowledges support from the Severo Ochoa Program (MINECO, Grant SEV-2013-0295). The authors acknowledge the assistance of B. Ballesteros in TEM imaging.
[1] S.-E. Park and T. R. Shrout, J. Appl. Phys. 82, 1804 (1997).

[2] G. Smolenskii, V. Isupov, N. Krainik, R. Pasinkov, and A. Sokolov, Ferroelectrics and Related Materials (Gordon and Breach, New York, 1984).

[3] L. E. Cross, Ferroelectrics 76, 241 (1987).

[4] A. Levstik, Z. Kutnjak, C. Filipič, and R. Pirc, Phys. Rev. B 57, 11204 (1998).

[5] R. Blinc, J. Dolinšek, A. Gregorovič, B. Zalar, C. Filipič, Z. Kutnjak, A. Levstik, and R. Pirc, Phys. Rev. Lett. 83, 424 (1999).

[6] V. Bobnar, Z. Kutnjak, R. A. Pirc, R. Blinc, and A. Levstik, Phys. Rev. Lett. 84, 5892 (2000).

[7] D. Viehland, S. J. Jang, L. E. Cross, and M. Wuttig, J. Appl. Phys. 68, 2916 (1990).

[8] B. Noheda, D. E. Cox, G. Shirane, S. E. Park, L. E. Cross, and Z. Zhong, Phys. Rev. Lett. 86, 3891 (2001).

[9] H. Fu and R. E. Cohen, Nature (London) 403, 281 (2000).

[10] G. Xu, P. M. Gehring, C. Stock, and K. Conlon, Phase Transitions 79, 135 (2006).

[11] G. Xu, J. Wen, C. Stock, and P. M. Gehring, Nat. Mater. 7, 562 (2008).

[12] G. Shirane, G. Y. Xu, and P. M. Gehring, Ferroelectrics 321, 7 (2005).

[13] P. Mokrý, A. K. Tagantsev, and N. Setter, Ferroelectrics 319, 209 (2005).

[14] M. F. Wong and K. Zeng, J. Am. Ceram. Soc. 94, 1079 (2011).

[15] M. F. Wong and K. Zeng, J. Appl. Phys. 107, 124104 (2010).
[16] X. Zhao, J. Dai, J. Wang, H. Chan, C. Choy, X. Wan, and H. Luo, Phys. Rev. B 72, 064114 (2005).

[17] V. V. Shvartsman and A. L. Kholkin, J. Appl. Phys. 101, 064108 (2007).

[18] A. Kholkin, A. Morozovska, D. Kiselev, I. Bdikin, B. Rodriguez, P. Wu, A. Bokov, Z.-G. Ye, B. Dkhil, L.-Q. Chen, M. Kosec, and S. V. Kalinin, Adv. Funct. Mater. 21, 1977 (2011).

[19] G. Xu, H. Hiraka, G. Shirane, and K. Ohwada, Appl. Phys. Lett. 84, 3975 (2004).

[20] E. H. Kisi and J. S. Forrester, J. Phys.: Condens. Matter 17, L381 (2005).

[21] M. Davis, D. Damjanovic, and N. Setter, J. Appl. Phys. 95, 5679 (2004).

[22] M. Davis, D. Damjanovic, and N. Setter, IEEE 14th International Symposium on Applications of Ferroelectrics, ISAF-04 (IEEE, Montreal, 2004), p. 205.

[23] M. Davis, D. Damjanovic, and N. Setter, IEEE 14th International Symposium on Applications of Ferroelectrics, ISAF-04 (IEEE, Montreal, 2004), p. 102.

[24] M. Iwata, K. Katsuraya, I. Suzuki, M. Maeda, N. Yasuda, and Y. Ishibashi, Jpn. J. Appl. Phys., Part 1 42, 6201 (2003).

[25] G. Catalan, J. Seidel, R. Ramesh, and J. F. Scott, Rev. Mod. Phys. 84, 119 (2012).

[26] P. S. I. Lukyanchuk, T. Nakajima, S. Okamura, J. F. Scott, and A. Gruverman, Nano Lett. 14, 6931 (2014).

[27] Huebner and Kampwirth, J. Low Temp. Phys. 15, 47 (1974).

[28] J. A. Cape and G. W. Lehman, J. Appl. Phys. 42, 5732 (1971).

[29] M. Seul and D. Andelman, Science 267, 476 (1995). 
[30] D. E. Cox, B. Noheda, G. Shirane, Y. Uesu, K. Fujishiro, and Y. Yamada, Appl. Phys. Lett. 79, 400 (2001).

[31] U. Pietsch, V. Holy, and T. Baumbach, High-Resolution $X$ ray Scattering: From Thin Films to Lateral Nanostructures (Springer-Verlag, New York, 2004).

[32] J. F. Scott, J. Chem. Phys. 48, 874 (1968).

[33] R. E. Cohen, Nature (London) 358, 136 (1992).

[34] A. Al-Zein, H. Dammak, P. Papet, O. Mathon, B. Hehlen, C. Levelut, J. Haines, and J. Rouquette, Inorg. Chem. 53, 3985 (2014).

[35] R. D. Shannon, J. Appl. Phys. 73, 348 (1993); Acta Cryst. A32, 751 (1976)

[36] X. Martí, P. Ferrer, J. Herrero-Albillos, J. Narvaez, V. Holy, N. Barrett, M. Alexe, and G. Catalan, Phys. Rev. Lett. 106, 236101 (2011).
[37] R. Jarrier, X. Marti, J. Herrero-Albillos, P. Ferrer, R. Haumont, P. Gemeiner, G. Geneste, P. Berthet, T. Schülli, P. Cevc et al., Phys. Rev. B 85, 184104 (2012).

[38] N. Domingo, J. Narvaez, M. Alexe, and G. Catalan, J. Appl. Phys. 113, 187220 (2013).

[39] C. J. M. Daumont, S. Farokhipoor, A. Ferri, J. C. Wojdeł, J. Íñiguez, B. J. Kooi, and B. Noheda, Phys. Rev. B 81, 144115 (2010).

[40] Z.-G. Ye, Handbook of Advanced Dielectric, Piezoelectric and Ferroelectric Materials, 1st ed. (Woodhead Publishing, Cambridge, MA, 2008).

[41] A. D. Rata, A. Herklotz, K. Nenkov, L. Schultz, and K. Dörr, Phys. Rev. Lett. 100, 076401 (2008).

[42] G. Burns and F. H. Dacol, Solid State Commun. 48, 853 (1983).

[43] J. Hlinka, J. Adv. Dielectr. 02, 1241006 (2012). 